\title{
Spatio-Temporal Pyramid Graph Convolutions for Human Action Recognition and Postural Assessment
}

\author{
K C Hemanth
}

\section{INTRODUCTION}

Recognition of human actions and associated interactions with objects and the environment is an important problem in computer vision due to its potential applications in a variety of domains. The most versatile methods can generalize to various environments and deal with cluttered backgrounds, occlusions, and viewpoint variations. Among them, methods based on graph convolutional networks that extract features from the skeleton have demonstrated promising performance. In this paper, we propose a novel Spatio-Temporal Pyramid Graph Convolutional Network (ST-PGN) for online action recognition for ergonomic risk assessment that enables the use of features from all levels of the skeleton feature hierarchy.

\section{REFERENCES}

[1] Gowda, S.N., 2017. Human activity recognition using combinatorial Deep Belief Networks. In Proceedings of the IEEE Conference on Computer Vision and Pattern Recognition Workshops (pp. 1-6).

[2] Wang, H. and Schmid, C., 2013. Action recognition with improved trajectories. In Proceedings of the IEEE international conference on computer vision (pp. 3551-3558).

[3] Jhuang, H., Gall, J., Zuffi, S., Schmid, C. and Black, M.J., 2013. Towards understanding action recognition. In Proceedings of the IEEE international conference on computer vision (pp. 3192-3199). [4] J. Carreira and A. Zisserman. Quo vadis, action recognition? a new model and the kinetics dataset. In proceedings of the IEEE Conference on Computer Vision and Pattern Recognition, pages 6299-6308, 2017. [5] T. Cloete and C. Scheffer. Benchmarking of a full-body inertial motion capture system for clinical gait analysis. In 2008 30th Annual International Conference of the IEEE Engineering in Medicine and Biology Society, pages 4579-4582. IEEE, 2008.

[6] A. Colim, P. Carneiro, N. Costa, P. M. Arezes, and N. Sousa. Ergonomic assessment and workstation design in a furniture manufacturing industrya case study. In Occupational and Environmental Safety and Health, pages 409-417. Springer, 2019.

[7] J. Dai, K. He, and J. Sun. Convolutional feature masking for joint object and stuff segmentation. In Proceedings of the IEEE Conference on Computer Vision and Pattern Recognition, pages 3992-4000, 2015. [8] Gowda, S.N. and Yuan, C., 2018, December. ColorNet: Investigating the importance of color spaces for image classification. In Asian Conference on Computer Vision (pp. 581-596). Springer, Cham.

[9] Y. Du, W. Wang, and L. Wang. Hierarchical recurrent neural network for skeleton based action recognition. In Proceedings of the IEEE conference on computer vision and pattern recognition, pages 1110-1118, 2015.

[10] X. Guo, J. Liu, and Y. Chen. Fitcoach: Virtual fitness coach empowered by wearable mobile devices. In IEEE INFOCOM 2017-IEEE Conference on Computer Communications, pages 1-9. IEEE, 2017.

[11] I. Habibie, W. Xu, D. Mehta, G. Pons-Moll, and C. Theobalt. In the wild human pose estimation using explicit $2 d$ features and intermediate $3 d$ representations. In Proceedings of the IEEE Conference on Computer Vision and Pattern Recognition, pages 10905-10914, 2019.

[12] Gowda, S.N., Rohrbach, M. and Sevilla-Lara, L., 2021, May. SMART Frame Selection for Action Recognition. In Proceedings of the AAAI Conference on Artificial Intelligence (Vol. 35, No. 2, pp. 1451-1459). 